\title{
THE PRIME RADICAL IN ALTERNATIVE RINGS
}

\author{
MICHAEL RICH
}

\begin{abstract}
The characterization by J. Levitzki of the prime radical of an associative ring $R$ as the set of strongly nilpotent elements of $R$ is adapted here to apply to a wide class of nonassociative rings. As a consequence it is shown that the prime radical is a hereditary radical for the class of alternative rings and that the prime radical of an alternative ring coincides with the prime radical of its attached Jordan ring.
\end{abstract}

In $1951 \mathrm{~J}$. Levitzki characterized the prime radical of an associative ring $R$ as the set of all elements $r \in R$ such that every $m$-sequence beginning with $r$ ends in zero [3]. An $m$-sequence was defined to be a sequence $\left\{a_{0}, a_{1}, \ldots\right.$, $\left.a_{n}, \ldots\right\}$ such that $a_{i} \in a_{i-1} R a_{i-1}$ for $i=1,2, \ldots$ Recently, C. Tsai [9] has given a similar characterization for the prime radical of a Jordan ring. Here we extend this characterization to the class of all $s$-rings and, as a consequence, are able to show that the prime radical is a hereditary radical on the class of alternative rings (i.e., if $A$ is an ideal of an alternative ring $R$, then $P(A)=A \cap P(R))$ and that $P(R)=P\left(R^{+}\right)$for all 2 and 3-torsion free alternative rings $R$. Although it is not known whether the prime radical is hereditary on the class of all $s$-rings, a partial result in this direction is obtained.

Recall that a not necessarily associative ring $R$ is called an $s$-ring for a positive integer $s$ if $A^{s}$ is an ideal of $R$ whenever $A$ is an ideal of $R\left(A^{s}\right.$ denotes the set of all sums of products $a_{1} a_{2} \cdots a_{s}$ for $a_{i} \in A$ under all possible associations). An ideal $P$ of $R$ is called a prime ideal if whenever $A_{1} A_{2} \cdots A_{s}$ $\subseteq P$ then $A_{i} \subseteq P$ for some $i$. Here $A_{1} A_{2} \cdots A_{s}$ denotes the product of the ideals under all possible associations. The prime radical, $P(R)$, of $R$ is the intersection of all prime ideals of $R$ and can be characterized as the set of all elements $r \in R$ such that every complementary system $M$ of $R$ which contains $r$ also contains 0 . A set $M$ in $R$ is a complementary system if whenever $A_{1}, A_{2}, \ldots, A_{s}$ are $s$ ideals of $R$ such that $A_{i} \cap M \neq \varnothing$ for $i=1,2, \ldots, s$, then $\left(A_{1} A_{2} \cdots A_{s}\right) \cap M \neq \varnothing[6]$, [8], [10].

To make this article self-contained we mention the following three properties of $P(R)$ which hold for any $s$-ring $R$. Proofs can be found in [6], [8] and [10].

(a) $P(R)=0$ if and only if $R$ contains no nonzero nilpotent ideals.

(b) $P(R / P(R))=0$.

Received by the editors May 29, 1975.

AMS (MOS) subject classifications (1970). Primary 17D05, 17E05.

Key words and phrases. Alternative ring, $s$-ring, prime radical, hereditary radical.

(1) American Mathematical Society 1976 
(c) $P(R)$ is the intersection of all ideals $Q$ of $R$ such that $R / Q$ contains no nonzero nilpotent ideals.

In what follows (a) denotes the principal ideal generated by $a$.

Definition. A sequence $\left\{a_{0}, a_{1}, \ldots, a_{n}, \ldots\right\}$ in an $s$-ring $R$ is called a $P$ sequence if $a_{n} \in\left(a_{n-1}\right)^{s}$ for $n=1,2, \ldots$ An element $a$ of $R$ is called strongly nilpotent if every $P$-sequence beginning with $a$ ends in zero.

THEOREM 1. In any s-ring $R$, the prime radical $P(R)$ consists of all strongly nilpotent elements of $R$.

Proof. Suppose that $a \in P(R)$ and that $M=\left\{a_{0}=a, a_{1}, \ldots, a_{n}, \ldots\right\}$ is a $P$-sequence beginning with $a$. Let $A_{1}, A_{2}, \ldots, A_{s}$ be ideals of $R$ such that $A_{i} \cap M \neq \varnothing$ for each $i$. Thus, there exist elements $a_{i_{1}} \in A_{1} \cap M, a_{i_{2}} \in$ $A_{2} \cap M, \ldots, a_{i_{s}} \in A_{s} \cap M$. Since by hypothesis $a_{i} \in\left(a_{i-1}\right)^{s}$, it follows that if $i \leqslant j$ then $\left(a_{j}\right) \subseteq\left(a_{i}\right)$. Let $t=\max \left(i_{1}, i_{2}, \ldots, i_{s}\right)$. Then $a_{t+1} \in\left(a_{t}\right)^{s} \subseteq$ $\left(a_{i_{1}}\right)\left(a_{i_{2}}\right) \cdots\left(a_{i_{s}}\right) \subseteq A_{1} A_{2} \cdots A_{s}$. We have shown that if $A_{i} \cap M \neq \varnothing$ for all $i$ then $\left(A_{1} A_{2} \cdots A_{s}\right) \cap M \neq \varnothing$. Thus $M$ is a complementary system. Since $a \in M \cap P(R)$ it follows that $0 \in M$. Thus $a_{l}=0$ for some $l$ and $a$ is a strongly nilpotent element.

Conversely, if $a \notin P(R)$ then there is a prime ideal $P$ such that $a \notin P$. Let $S$ be the sequence $\left\{a_{0}, a_{1}, \ldots, a_{n}, \ldots\right\}$ where $a_{0}=a, a_{1} \in\left(a_{0}\right)^{s} \cap C(P)$, $\ldots, a_{n} \in\left(a_{n-1}\right)^{s} \cap C(P), \ldots$. Such a sequence can be defined inductively since if $a_{n-1}$ has been chosen in $C(P)$ then $\left(a_{n-1}\right)^{s} \nsubseteq P$, so that there exists $a_{n} \in\left(a_{n-1}\right)^{s} \cap C(P)$. Thus, $S$ is a $P$-sequence beginning in $a$ which does not end in zero. It follows that $a$ is not strongly nilpotent. We conclude that $P(R)$ is precisely the set of strongly nilpotent elements of $R$.

It is well known that the prime radical is hereditary on associative rings; i.e., if $R$ is an associative ring and if $A$ is an ideal of $R$ then $P(A)=A \cap P(R)$ [1]. We proceed to investigate this concept for $s$-rings in general.

DEFINITION. A ring $R$ is called a strongly $s$-ring if every subring of $R$ is an $s$-ring.

Clearly every variety all of whose members are $s$-rings is a variety of strongly $s$-rings. Thus, the varieties of associative, alternative and Lie rings are strongly 2-varieties and the varieties of Jordan and standard rings are strongly 3-varieties [6].

LemMA. If $R$ is a strongly s-ring and if $B$ is a subring of $R$ then $B \cap P(R)$ $\subseteq P(B)$.

Proof. Since $B$ is an $s$-ring, $P(B)$ is the set of strongly nilpotent elements in $B$. Let $b \in B \cap P(R)$. Then any $P$-sequence of $R$ beginning with $b$ ends in zero. Let $S$ be a $P$-sequence of $B$ beginning with $b$. Then $S$ is clearly a $P$ sequence of $R$ beginning with $b$. Thus $S$ ends in zero so that $b \in P(B)$.

THEOREM 2. The prime radical is hereditary on alternative rings $R$ (i.e., if $A$ is an ideal of $R$ then $P(A)=A \cap P(R)$ ).

Proof. Since $R$ is a strongly 2-ring it follows by the Lemma that $A \cap P(R)$ $\subseteq P(A)$. Now Slater [7, Theorem $C$ ] has shown that if $R$ contains no nilpotent ideals then $A$ (as a ring) contains no nilpotent ideals. This translates to " $P(R)$ $=0$ implies $P(A)=0$ " and is sufficient to prove the converse. For, suppose 
that $P(R) \neq 0$. Then, by (b), $P(R / P(R))=0$. Thus, by Slater's result it follows that $P((A+P(R)) / P(R) \cong P(A /(A \cap P(R)))=0$. Thus

$$
A /(A \cap P(R))
$$

contains no nonzero nilpotent ideals. Since $P(R)$ can be characterized as the intersection of all ideals $Q$ of $R$ such that $R / Q$ contains no nonzero nilpotent ideals [6], it follows immediately that $P(A) \subseteq A \cap P(R)$.

REMARKs.1. A property $P$ of rings is called a radical property in the sense of Kurosh if: (a) homomorphic images of $P$-rings are $P$-rings, (b) every ring $R$ contains a $P$-ideal $S$ which contains every $P$-ideal, and (c) $R / S$ contains no nonzero $P$-ideals. It is known that the prime radical is a radical property for associative rings [ 1, p. 57]. In view of the fact that the prime radical coincides with the Baer lower radical in any $s$-ring [6], and the fact that in any alternative ring $R, P(R)=0$ implies $P(A)=0$ for all ideals $A$ of $R$, we may observe that the proof in [1] that the prime radical is a radical property for associative rings can be adopted word for word to show that the prime radical is a radical property for alternative rings. Note also, by virtue of the proof of Theorem 2, that in any strongly $s$-ring in which $P(R)=0$ implies $P(A)=0$ for all ideals $A$ of $R$, the prime radical is hereditary. I do not know if this is the case for Jordan and Lie rings.

2 . In view of the fact that in an $s$-ring $R$ prime ideals are defined in terms of the number $s$, it is necessary to show that if $R$ is an $s$-ring and also a $t$-ring for $s \neq t$, that $P(R)$ is unchanged whether $R$ is viewed as an $s$-ring or as a $t$ ring. However, the characterization of $P(R)$ used in the proof of Theorem 2 as the intersection of all ideals $Q$ of $R$ such that $R / Q$ contains no nonzero nilpotent ideals does the trick, since nilpotent ideals are not defined in terms of the number $s$.

If $R$ is an alternative ring the attached ring $R^{+}$of $R$ is setwise equal to $R$ but multiplication in $R^{+}$is given by $a \cdot b=a b+b a$ where $a b$ is the multiplication in $R$. It is well known that if $R$ is alternative then $R^{+}$is a special Jordan ring. Thus it is of interest to relate the radical properties of $R$ to those of $R^{+}$. Using the structure theory for simple alternative rings, it is easy to see that $R$ is simple if and only if $R^{+}$is simple. McCrimmon [5] has shown that $J(R)=J\left(R^{+}\right)$where $J$ denotes the Jacobson radical. The following theorem treats the same problem for the prime radical and generalizes the result of Erickson and Montgomery [2] who obtained the result for associative rings. We shall say that $R$ is $n$-torsion free if $n r=0$ for $r \in R$ implies $r=0$.

THEOREM 3. If $R$ is $a 2$ and 3-torsion free alternative ring then $P(R)=P\left(R^{+}\right)$.

Proof. Since $R^{+}$is a Jordan ring it is a strongly 3-ring. By $[a]$ we denote the principal ideal generated by $a$ in $R^{+}$and by $[a]^{n}$ we always mean multiplication in $R^{+}$. Let $a \in P(R)$. Then any sequence $\left\{a, a_{1}, \ldots, a_{n}, \ldots\right\}$ where $a_{1} \in(a)^{2}$ and $a_{i} \in\left(a_{i-1}\right)^{2}$ for all $i$ ends in zero. Let $S=\left\{a=b_{0}, b_{1}, \ldots\right.$, $\left.b_{n}, \ldots\right\}$ be a $P$-sequence of $R^{+}$beginning in $a$. Thus, $b_{i} \in\left[b_{i-1}\right]^{3}$ for all $i$. However $\left[b_{i-1}\right]^{3} \subseteq\left[b_{i-1}\right]^{2} \subseteq\left(b_{i-1}\right)^{2}$ for all $i$. Thus $S$ is a $P$-sequence of $R$ beginning with $a$, from which it follows that $0 \in S$. Hence $a$ is a strongly nilpotent element of $R^{+}$and by Theorem 1, $a \in P\left(R^{+}\right)$. Thus, $P(R)$ $\subseteq P\left(R^{+}\right)$. 
For the converse it is sufficient to show that $P(R)=0$ implies $P\left(R^{+}\right)=0$. For, under this assumption, if $P(R) \neq 0$ then we consider $P(R / P(R))$. Since $P(R / P(R))=0$ we get $P\left((R / P(R))^{+}\right)=0$. But $(R / P(R))^{+} \cong R^{+} / P(R)^{+}$ $=R^{+} / P(R)$. Thus $P\left(R^{+} / P(R)\right)=0$ from which it follows that $R^{+} / P(R)$ contains no nonzero nilpotent ideals. But $P\left(R^{+}\right)$is the intersection of all ideals $Q$ of $R^{+}$such that $R^{+} / Q$ contains no nonzero nilpotent ideals. Thus $P\left(R^{+}\right) \subseteq P(R)$.

It remains to show that $P(R)=0$ implies that $P\left(R^{+}\right)=0$. But if $P\left(R^{+}\right)$ $\neq 0$ then there exists a nonzero nilpotent ideal of $R^{+}$. Since $R^{+}$is a 3-ring, we can assume without loss of generality that there exists a nonzero ideal $U$ of $R^{+}$ such that $\left(U^{+}\right)^{3}=0\left(\left(U^{+}\right)^{3}\right.$ denotes the 3rd-power of $U$ in the ring $\left.R^{+}\right)$. Let $u \in U, r \in R$. Then $v=u^{2} \cdot r \in U$ and since $\left(U^{+}\right)^{3}=0$ it follows that $u^{2} \cdot v=0$. But

$$
u^{2} \cdot v=u^{2}\left(u^{2} r+r u^{2}\right)+\left(u^{2} r+r u^{2}\right) u^{2}=u^{4} r+2 u^{2} r u^{2}+r u^{4}
$$

since $R$ is alternative. But $u^{4}=0$ and $R$ is 2 -torsion free. Thus, we have $u^{4}=u^{2} r u^{2}=0$ for all $r \in R$. Since $R$ is 3-torsion free and $P(R)=0$, a result of Kleinfeld [4, Lemma 1] yields $u^{2}=0$ for all $u \in U$. Therefore $w \cdot u=0$ for all $w, u \in U$. Let $u \in U$ and $w=u \cdot r$ for $r \in R$. Then $w \in U$ so that $u \cdot w=0$. But $u \cdot w=u^{2} r+r u^{2}+2 u r u$. Since $R$ is 2-torsion free we arrive at $u^{2}=u R u=0$ for all $u \in U$. A second application of [4, Lemma 1] yields $U=0$ which is a contradiction. Thus $P(R)=0$ implies $P\left(R^{+}\right)=0$ so that, in general, $P\left(R^{+}\right) \subseteq P(R)$. This completes the proof.

REMARK. If $R$ is an associative ring and $L(R)$ its attached Lie ring, then a similar application of Theorem 1 as that used in the beginning of the proof of Theorem 3 shows that $P(R) \subseteq P(L(R))$. In general however, $P(R)$ $\neq P(L(R))$. For if $R$ is a commutative ring then $L(R)$ is an abelian Lie ring. Therefore $L(R)$ contains no prime ideals so that $P(L(R))=R$. On the other hand $P(R)$ consists of the set of nilpotent elements of $R$. Thus, if $R$ is not a nil ring then $P(R) \varsubsetneqq P(L(R))$. However, $R$ always contains a subring $A$ such that $P(R)=P(L(A))$. In fact one can always choose $A=P(R)$. For clearly $P(L(P(R))) \subseteq P(R)$. On the other hand, if $r \in P(R)$ then $r$ is strongly nilpotent in $R$, hence strongly nilpotent in $L(R)$. Moreover, since $r \in P(R)$ then $r$ is strongly nilpotent in $L(P(R))$. Therefore, $r \in P(L(P(R)))$ and it follows that $P(R)=P(L(P(R)))$.

\section{REFERENCES}

1. N. J. Divinsky, Rings and radicals, Math. Expositions, no. 14, Univ. of Toronto Press, Toronto, Ont., 1965. MR 33 \# 5654.

2. T. S. Erickson and S. Montgomery, The prime radical in special Jordan rings, Trans. Amer. Math. Soc. 156 (1971), $155-164$. MR 43 \# 306.

3. J. Levitski, Prime ideals and the lower radical, Amer. J. Math. 73 (1951), 25-29. MR 12, 474.

4. E. Kleinfeld, Alternative nil rings, Ann. of Math. (2) 66 (1957), 395-399. MR 19, 383.

5. K. McCrimmon, A characterization of the Jacobson-Smiley radical, J. Algebra 18 (1971), 565-573. MR 43 \#3318.

6. M. Rich, Some radical properties of s-rings, Proc. Amer. Math. Soc. 30 (1971), 40-42. MR 44 \#267.

7. M. Slater, Ideals in semiprime alternative rings, J. Algebra 8 (1968), 60-76. MR 38 \#2183. 
8. C. Tsai, The prime radical in a Jordan ring, Proc. Amer. Math. Soc. 19 (1968), 1171-1175. MR 37 \# 6336.

9. - An internal characterization of the prime radical of a Jordan algebra, Proc. Amer. Math. Soc. 36 (1972), 361 -364. MR 47 \# 1898.

10. P. J. Zwier, Prime ideals in a large class of nonassociative rings, Trans. Amer. Math. Soc. 158 (1971), 257-271. MR 43 \# 7478.

Department of Mathematics, Temple University,Philadelphia, Pennsylvania 19122 (Current address)

Department of Mathematics, Ben Gurion University of the Negev, Beer Sheva, Israel 